\title{
Dissipative Output Tracking Control of Linear Systems with Time Delay
}

\author{
Yi Zhang, ${ }^{1,2}$ Yile Zhang, ${ }^{2,3}$ Jinghao Li, ${ }^{2}$ and Baoyan $\mathrm{Zhu}^{4}$ \\ ${ }^{1}$ School of Science, Shenyang University of Technology, Shenyang, Liaoning 110870, China \\ ${ }^{2}$ Institute of Systems Science, Northeastern University, Shenyang, Liaoning 110819, China \\ ${ }^{3}$ Department of Mathematical and Statistical Sciences, University of Alberta, 116 Street and 85 Avenue, \\ Edmonton, AB, Canada T6G $2 G 1$ \\ ${ }^{4}$ College of Science, Shenyang JianZhu University, Shenyang, Liaoning 110168, China
}

Correspondence should be addressed to Jinghao Li; jhlee_neu@yeah.net

Received 28 February 2013; Accepted 29 April 2013

Academic Editor: Guanglu Zhou

Copyright (C) 2013 Yi Zhang et al. This is an open access article distributed under the Creative Commons Attribution License, which permits unrestricted use, distribution, and reproduction in any medium, provided the original work is properly cited.

\begin{abstract}
The problem of dissipative output tracking control of linear systems with time delay is investigated. Firstly, an augmented system is constructed to describe dissipative output tracking control error, and the concept of dissipative output tracking is defined. Based on this, some sufficient conditions are derived in terms of linear matrix inequalities (LMIs) technique, which ensure that the augmented system is dissipative and stable; then design methods of dissipative output tracking state-feedback controller are provided, and the desired controller gain can be expressed through the solutions of LMIs. Finally, a numerical simulation example is given to illustrate the validity of the proposed results.
\end{abstract}

\section{Introduction}

Output tracking control is one of the most fundamental and important control problems in control theory and applications. The essence of output tracking control is to enable controlled output to approach reference output as closely as possible. Output tracking control has been applied successfully to many fields, such as aircraft [1], robot [2-4], and biomathematics [5]. Meanwhile, many control methods have been developed in this area to analyze and control systems. The authors in [6] have studied the adaptive asymptotic tracking problems for a class of large-scale systems. Many results on the fuzzy adaptive tracking control problems have been obtained in [7-9]. By using a sliding-mode controller/ observer scheme, the authors in [10] have solved robust output tracking problems. In [11], the authors have investigated output tracking problem for uncertain nonlinear systems. Robust output tracking control problem has been discussed for time-delay nonlinear systems by neural network in [12]. $H_{\infty}$ output tracking control has been studied by T-S fuzzy model approach in [13]. [14] has studied $H_{\infty}$ output tracking problems for network-based control systems. The chaotic tracking control has been first investigated for SIR epidemic model in [15]; a tracking control method is given to control chaotic motion in the epidemic model, and the corresponding state feedback controllers are designed such that the density of infected individuals can converge to zero. In [16], the tracking control of chaos is studied for singular biological economy systems; a controller is designed which guarantees that the output of chaos system can track an expected constant value or period orbit. In order to eliminate the chaos or Neimark-Sacker bifurcation of the discrete epidemic model, a tracking controller is designed in [17] such that the disease disappears gradually. Different new results about output tracking control have been obtained in these literatures which provide us significant and useful control approaches.

Notion of dissipativity was introduced by Willems in 1970s [18, 19], which can be regarded as a generalization of $H_{\infty}$ performance as well as positive realness performances and passivity. Essentially, the practical interpretation of dissipativity is that there exists a nonnegative storage function such that energy consumption rate is less than a given energy 
supply rate. The representative researches of dissipative theory for linear systems can be found in [20-22]. However, to the best of our knowledge, there are no research on output tracking control problems from the perspective of dissipativity. All of these motivate us to achieve the current study.

In this paper, dissipative output tracking control problem is formulated for linear time-delay systems. From the view of dissipativity, the process of output tracking control can be regarded as the process of energy dissipation. Dissipative output tracking control generalizes some previous results about output tracking control, including $H_{\infty}$ output tracking control, whose concept is first presented for linear time-delay systems in this paper. Then, some sufficient conditions are derived such that the considered systems are dissipative and stable by employing LMIs technique. Following these results, dissipative output tracking controllers are designed which also ensure the resultant closed-loop systems to be stable. Compared with some existing results, this paper provides a new way to solve output tracking control problems for linear time-delay systems.

This paper is organized as follows. Dissipative output tracking control problem is formulated in Section 2. By using PI control method, the output tracking control problem of the original system is transformed into the dissipative analysis problem of an augmented system. In Section 3, some sufficient conditions are explored for the augmented system with and without time delay to be dissipative, and then the corresponding dissipative output tracking controllers are designed. In Section 4, we bring a demonstrative example to show the effectiveness of the proposed method. Some conclusions are drawn and some potential research interests are stated in Section 5 .

\section{Problem Formulation}

Consider the following linear time-delay system:

$$
\begin{gathered}
\dot{x}(t)=A x(t)+A_{d} x(t-d)+B u(t), \\
y(t)=C x(t)+C_{d} x(t-d)+D u(t), \\
x(t)=\eta(t), \quad t \in[-d, 0],
\end{gathered}
$$

where $x(t) \in \mathbb{R}^{n}$ is state vector, $u(t) \in \mathbb{R}^{m}$ is input vector, $y(t) \in \mathbb{R}^{l}$ is output vector, and the initial condition $\eta(t)$ is a continuously differentiable vector-valued function over $[-d, 0] . A, A_{d}, B, C, C_{d}$, and $D$ are real constant matrices with appropriate dimensions. Sometimes we denote this system by $\left(A, A_{d}, B, C, C_{d}, D\right)$ for simplicity.

In practical world, it is often expected that the output vector $y(t)$ approaches a desired reference output vector $y_{\text {ref }}(t)$ asymptotically which may be produced from a reference system of the form

$$
\begin{aligned}
& \dot{x}_{\text {ref }}(t)=A_{\text {ref }} x_{\text {ref }}(t) \\
& y_{\text {ref }}(t)=C_{\text {ref }} x_{\text {ref }}(t),
\end{aligned}
$$

where $x_{\text {ref }}(t) \in \mathbb{R}^{n}$ is reference state vector, $y_{\text {ref }}(t) \in \mathbb{R}^{l}$ is reference output vector. $A_{\text {ref }}$ and $C_{\text {ref }}$ are real constant matrices with appropriate dimensions. The response of this reference system exists uniquely for all given initial conditions.

To this end, it is required that

$$
\lim _{t \rightarrow \infty}\left(y(t)-y_{\text {ref }}(t)\right)=0 .
$$

Let $e(t)=y(t)-y_{\text {ref }}(t)$ be error vector, then (3) is equivalent to $\lim _{t \rightarrow \infty} e(t)=0$. Using the well-known PI control approach, we introduce the following error integrator model:

$$
\begin{aligned}
\dot{q}(t) & =e(t)=y(t)-y_{\mathrm{ref}}(t) \\
& =C x(t)+C_{d} x(t-d)+D u(t)-y_{\mathrm{ref}}(t) .
\end{aligned}
$$

From (1) and (4), the following augmented system is constructed:

$$
\begin{gathered}
\dot{x}_{L}(t)=A_{L} x_{L}(t)+A_{L d} x_{L}(t-d)+B_{L} u(t)-N_{L} y_{\text {ref }}(t) \\
e(t)=C_{L} x_{L}(t)+C_{L d} x_{L}(t-d)+D u(t)-y_{\text {ref }}(t) \\
x_{L}(t)=\left[\begin{array}{c}
\eta(t) \\
0
\end{array}\right], \quad t \in[-d, 0],
\end{gathered}
$$

where $x_{L}^{\mathrm{T}}(t)=\left[\begin{array}{ll}x^{\mathrm{T}}(t) & q^{\mathrm{T}}(t)\end{array}\right]$ and

$$
\begin{gathered}
A_{L}=\left[\begin{array}{ll}
A & 0 \\
C & 0
\end{array}\right], \quad A_{L d}=\left[\begin{array}{ll}
A_{d} & 0 \\
C_{d} & 0
\end{array}\right], \quad B_{L}=\left[\begin{array}{c}
B \\
D
\end{array}\right], \\
N_{L}=\left[\begin{array}{l}
0 \\
I
\end{array}\right], \quad C_{L}=\left[\begin{array}{ll}
C & 0
\end{array}\right], \quad C_{L d}=\left[\begin{array}{ll}
C_{d} & 0
\end{array}\right] .
\end{gathered}
$$

In order to make the output vector $y(t)$ approaches the reference output vector $y_{\text {ref }}(t)$ asymptotically, the following concept is introduced for system (5) from the view of dissipativity.

Definition 1. For system (5) with a given supply rate $r\left(y_{\text {ref }}(t)\right.$, $e(t)$ ), if there exists a nonnegative storage function $V(x(t))$ with $V(0)=0$ such that the following dissipative inequality

$$
\dot{V}(x(t)) \leq r\left(y_{\text {ref }}(t), e(t)\right), \quad \forall t \geq 0
$$

holds for all the $x(t), y_{\text {ref }}(t)$, and $e(t)$, then system (5) is said to be dissipative. If the dissipative inequality (7) is strict, system (5) is said to be strictly dissipative. Furthermore, system (1) is said to be (strictly) dissipative output tracking in case of system (5) being (strictly) dissipative.

Introduce the supply rate function associated with system (5) which has the following quadratic form:

$$
\begin{aligned}
r\left(y_{\text {ref }}(t), e(t)\right)= & \langle e(t), Q e(t)\rangle+2\left\langle e(t), S y_{\text {ref }}(t)\right\rangle \\
& +\left\langle y_{\text {ref }}(t), R y_{\text {ref }}(t)\right\rangle,
\end{aligned}
$$

where $Q=Q^{\mathrm{T}} \in \mathbb{R}^{l \times l}, R=R^{\mathrm{T}} \in \mathbb{R}^{l \times l}$, and $S \in \mathbb{R}^{l \times l}$ are real matrices with appropriate dimensions, and $\langle a, b\rangle=a^{\mathrm{T}} b$, for all $a, b \in \mathbb{R}^{l}$.

We first present the following lemmas which will be used later. 
Lemma 2. Assume system that $(A, B)$ is controllable. Then augmented system $\left(A_{L}, B_{L}\right)$ is controllable if and only if

$$
\operatorname{rank}\left[\begin{array}{cc}
A & B \\
C & D
\end{array}\right]=n+l \text {. }
$$

Proof. First let $Q_{L}=\left[\begin{array}{llllll}B_{L} & A_{L} B_{L} & A_{L}^{2} B_{L} & \cdots & A_{L}^{n-1} B_{L}\end{array}\right]$. Then

$$
\begin{aligned}
\operatorname{rank} Q_{L} & =\operatorname{rank}\left[\begin{array}{ccccc}
B & A B & A^{2} B & \cdots & A^{n-1} B \\
D & C B & C A B & \cdots & C A^{n-2} B
\end{array}\right] \\
& =\operatorname{rank}\left[\begin{array}{cc}
A & B \\
C & D
\end{array}\right]\left[\begin{array}{cc}
0 & Q \\
I & 0
\end{array}\right],
\end{aligned}
$$

where $Q=\left[\begin{array}{lllll}B & A B & A^{2} B & \cdots & A^{n-1} B\end{array}\right]$. Therefore, we can conclude from rank $Q=n$ that this result is true. This completes the proof.

Remark 3. It is noted that Lemma 2 gives a necessary and sufficient condition for the augmented system to be controllable. As we know, if a system is controllable, then the eigenvalues of the system can be assigned arbitrarily, which implies that the system can be stabilized. This lemma explains the relationship that, under appropriate condition, the stabilizability of the original system can ensure the stabilizability of the augmented system, which has a potential application in controller design.

Lemma 4 (Schur complement [23]). For a given symmetric matrix $S \in \mathbb{R}^{n \times n}$, and $S=\left[\begin{array}{ll}S_{11} & S_{12} \\ S_{12}^{T} & S_{22}\end{array}\right]$, where $S_{i j} \in \mathbb{R}^{n_{i} \times n_{j}}$ are matrix blocks with appropriate dimensions, the following statements are equivalent:

(1) $S<0$;

(2) $S_{11}<0, S_{22}-S_{12}^{\mathrm{T}} S_{11}^{-1} S_{12}<0$;

(3) $S_{22}<0, S_{11}-S_{12} S_{22}^{-1} S_{12}^{\mathrm{T}}<0$.

\section{Main Results}

Consider the following linear time-delay system:

$$
\begin{gathered}
\dot{x}_{L}(t)=A_{L} x_{L}(t)+A_{L d} x_{L}(t-d)-N_{L} y_{\text {ref }}(t) \\
e(t)=C_{L} x_{L}(t)+C_{L d} x_{L}(t-d)-y_{\text {ref }}(t) \\
x_{L}(t)=\left[\begin{array}{c}
\eta(t) \\
0
\end{array}\right], \quad t \in[-d, 0],
\end{gathered}
$$

where $x_{L}(t) \in \mathbb{R}^{n}$ is augmented state vector, $y_{\text {ref }}(t) \in \mathbb{R}^{l}$ is reference output vector, $u(t) \in \mathbb{R}^{m}$ is input vector, $e(t) \in \mathbb{R}^{l}$ is error output vector, and $A_{L}, A_{L d}, C_{L}, C_{L d}$, and $N_{L}$ are known constant matrices with appropriate dimensions.

Then, select the following Lyapunov functional as storage function

$$
V\left(x_{L}(t), t\right)=x_{L}^{\mathrm{T}}(t) P x_{L}(t)+\int_{t-d}^{t} x_{L}^{\mathrm{T}}(\tau) V x_{L}(\tau) d \tau,
$$

where $0 \leq P \in \mathbb{R}^{n \times n}, 0<V \in \mathbb{R}^{n \times n}$.

Consider the supply rate (8), then for system (11), we have the following results.
Theorem 5. For the time-delay system (11) and the given supply rate (8), if the following LMIs

$$
\begin{gathered}
P=P^{\mathrm{T}} \geq 0, \\
V>0, \\
{\left[\begin{array}{ccc}
A_{L}^{\mathrm{T}} P+P A_{L}+V-C_{L}^{\mathrm{T}} Q C_{L} & -P N_{L}+C_{L}^{\mathrm{T}} \mathrm{Q}-C_{L}^{\mathrm{T}} S & P A_{L d}-C_{L}^{\mathrm{T}} Q C_{L d} \\
* & -\mathrm{Q}+S+S^{\mathrm{T}}-R & \mathrm{QC} C_{L d}-S^{\mathrm{T}} C_{L d} \\
* & * & -V-C_{L d}^{\mathrm{T}} Q C_{L d}
\end{array}\right] \leq 0}
\end{gathered}
$$

have feasible solutions $P, V$, then the linear time-delay system (11) is dissipative. Moreover, if LMIs (13) are strict, then system (11) is strictly dissipative; in this case when $Q<0$, system (11) is also stable. The "*" in LMIs denotes the terms that can be induced by symmetric block in a matrix.

Proof. Combine system (11) with the storage function (12), then the time derivative of $V\left(x_{L}(t), t\right)$ along the trajectory of system (11) is given as

$$
\begin{aligned}
\dot{V}( & \left.x_{L}(t), t\right) \\
= & \dot{x}_{L}^{\mathrm{T}}(t) P x_{L}(t)+x_{L}^{\mathrm{T}}(t) P \dot{x}_{L}(t) \\
= & \left(A_{L} x_{L}(t)+A_{L d} x_{L}(t-d)+N_{L} y_{\text {ref }}(t)\right)^{\mathrm{T}} P x_{L}(t) \\
& +x_{L}^{\mathrm{T}}(t) P\left(A_{L} x_{L}(t)+A_{L d} x_{L}(t-d)-N_{L} y_{\text {ref }}(t)\right) \\
& +x_{L}^{\mathrm{T}}(t) V x_{L}(t)-x_{L}^{\mathrm{T}}(t-d) V x_{L}(t-d) .
\end{aligned}
$$

Associated with the supply rate (8), we have

$$
\begin{aligned}
\dot{V}\left(x_{L}\right. & (t), t)-r\left(y_{\mathrm{ref}}(t), e(t)\right) \\
= & \left(A_{L} x_{L}(t)+A_{L d} x_{L}(t-d)-N_{L} y_{\mathrm{ref}}(t)\right)^{\mathrm{T}} P x_{L}(t) \\
& +x_{L}^{\mathrm{T}}(t) P\left(A_{L} x_{L}(t)+A_{L d} x_{L}(t-d)-N_{L} y_{\mathrm{ref}}(t)\right) \\
& +x_{L}^{\mathrm{T}}(t) V x_{L}(t)-x_{L}^{\mathrm{T}}(t-d) V x_{\mathrm{L}}(t-d) \\
& -\left(C_{L} x_{L}(t)+C_{L d} x_{L}(t-d)-y_{\mathrm{ref}}(t)\right)^{\mathrm{T}} \\
& \times Q\left(C_{L} x_{L}(t)+C_{L d} x_{L}(t-d)-y_{\mathrm{ref}}(t)\right) \\
& -2\left(C_{L} x_{L}(t)+C_{L d} x_{L}(t-d)-y_{\mathrm{ref}}(t)\right)^{\mathrm{T}} S y_{\mathrm{ref}}(t) \\
& -y_{\mathrm{ref}}^{\mathrm{T}}(t) R y_{\mathrm{ref}}(t) \\
= & \left(A_{L} x_{L}(t)+A_{L d} x_{L}(t-d)-N_{L} y_{\mathrm{ref}}(t)\right)^{\mathrm{T}} P x_{L}(t) \\
& +x_{L}^{\mathrm{T}}(t) P\left(A_{L} x_{L}(t)+A_{L d} x_{L}(t-d)-N_{L} y_{\mathrm{ref}}(t)\right) \\
& +x_{L}^{\mathrm{T}}(t) V x_{L}(t)-x_{L}^{\mathrm{T}}(t-d) V x_{L}(t-d) \\
& -\left(C_{L} x_{L}(t)+C_{L d} x_{L}(t-d)-y_{\mathrm{ref}}(t)\right)^{\mathrm{T}}
\end{aligned}
$$




$$
\begin{aligned}
& \quad \times Q\left(C_{L} x_{L}(t)+C_{L d} x_{L}(t-d)-y_{\mathrm{ref}}(t)\right) \\
& -\left(C_{L} x_{L}(t)+C_{L d} x_{L}(t-d)-y_{\mathrm{ref}}(t)\right)^{\mathrm{T}} S y_{\mathrm{ref}}(t) \\
& -y_{\mathrm{ref}}^{\mathrm{T}}(t) S^{\mathrm{T}}\left(C_{L} x_{L}(t)+C_{L d} x_{L}(t-d)-y_{\mathrm{ref}}(t)\right) \\
& -y_{\mathrm{ref}}^{\mathrm{T}}(t) R y_{\mathrm{ref}}(t) \\
& =\left[\begin{array}{c}
x_{L}(t) \\
y_{\mathrm{ref}}(t) \\
x_{L}(t-d)
\end{array}\right]^{\mathrm{T}} J\left[\begin{array}{c}
x_{L}(t) \\
y_{\mathrm{ref}}(t) \\
x_{L}(t-d)
\end{array}\right]
\end{aligned}
$$

where

$$
J=\left[\begin{array}{ccc}
A_{L}^{\mathrm{T}} P+P A_{L}+V-C_{L}^{\mathrm{T}} Q C_{L} & -P N_{L}+C_{L}^{\mathrm{T}} Q-C_{L}^{\mathrm{T}} S & P A_{L d}-C_{L}^{\mathrm{T}} Q C_{L d} \\
* & -Q+S+S^{\mathrm{T}}-R & Q C_{L d}-S^{\mathrm{T}} C_{L d} \\
* & * & -V-C_{L d}^{\mathrm{T}} Q C_{L d}
\end{array}\right]
$$

Therefore, if LMIs (13) have feasible solutions $P$ and $V$, it is from Definition 1 that linear time-delay system (11) is dissipative.

Next, we show the stability of system (11). To this end, assume that system (11) is strictly dissipative, then we separate $J$ into

$$
\begin{aligned}
J= & {\left[\begin{array}{ccc}
A_{L}^{\mathrm{T}} P+P A_{L}+V & -P N_{L}+C_{L}^{\mathrm{T}} \mathrm{Q}-C_{L}^{\mathrm{T}} S & P A_{L d} \\
* & -Q+S+S^{\mathrm{T}}-R & Q C_{L d}-S^{\mathrm{T}} C_{L d} \\
* & * & -V
\end{array}\right] } \\
& +\left[\begin{array}{ccc}
-C_{L}^{\mathrm{T}} Q C_{L} & 0 & -C_{L}^{\mathrm{T}} Q C_{L d} \\
* & 0 & 0 \\
* & * & -C_{L d}^{\mathrm{T}} \mathrm{Q} C_{L d}
\end{array}\right]<0 .
\end{aligned}
$$

Note that

$$
\left[\begin{array}{ccc}
-C_{L}^{\mathrm{T}} Q C_{L} & 0 & -C_{L}^{\mathrm{T}} Q C_{L d} \\
* & 0 & 0 \\
* & * & -C_{L d}^{\mathrm{T}} Q C_{L d}
\end{array}\right]=-\left[\begin{array}{c}
C_{L}^{\mathrm{T}} \\
0 \\
C_{L d}^{\mathrm{T}}
\end{array}\right] Q\left[\begin{array}{lll}
C_{L} & 0 & C_{L d}
\end{array}\right]
$$

This together with $Q<0$ yields

$$
J=\left[\begin{array}{cccc}
A_{L}^{\mathrm{T}} P+P A_{L}+V & -P N_{L}+C_{L}^{\mathrm{T}} \mathrm{Q}-C_{L}^{\mathrm{T}} S & P A_{L d} & C_{L}^{\mathrm{T}} \\
* & -\mathrm{Q}+S+S^{\mathrm{T}}-R & \mathrm{Q} C_{L d}-S^{\mathrm{T}} C_{L d} & 0 \\
* & * & -V & C_{L d}^{\mathrm{T}} \\
* & * & * & Q^{-1}
\end{array}\right]<0 .
$$

It is easy to see that the following LMI holds:

$$
\widehat{J}=\left[\begin{array}{cc}
A_{L}^{\mathrm{T}} P+P A_{L}+V & P A_{L d} \\
* & -V
\end{array}\right]<0 .
$$

Then, by the Lyapunov stability theory in [24], it can be seen that system (11) is stable. This completes the proof.
In fact, when the time-delay is absent, the augmented system (11) reduces to the following autonomous system:

$$
\begin{gathered}
\dot{x}_{L}(t)=A_{L} x_{L}(t)-N_{L} y_{\text {ref }}(t), \\
e(t)=C_{L} x_{L}(t)-y_{\text {ref }}(t) .
\end{gathered}
$$

In this case, choose the Lyapunov function of the following form as the storage function

$$
V(x(t), t)=x^{\mathrm{T}}(t) P x(t),
$$

where $0 \leq P \in \mathbb{R}^{n \times n}$

Then, we have the following result for system (21).

Theorem 6. For system (21) and the given supply rate (8), if the following LMIs

$$
P=P^{\mathrm{T}} \geq 0
$$

$$
\left[\begin{array}{cc}
A_{L}^{\mathrm{T}} P+P A_{L}-C_{L}^{\mathrm{T}} Q C_{L} & -P N_{L}+C_{L}^{\mathrm{T}} \mathrm{Q}-C_{L}^{\mathrm{T}} S \\
-N_{L}^{\mathrm{T}} P+Q C_{L}-S^{\mathrm{T}} C_{L} & -Q+S+S^{\mathrm{T}}-R
\end{array}\right] \leq 0
$$

have a feasible solution $P$, then the tracking system (21) is dissipative. Moreover, if LMIs (23) are strict, then system (21) is strictly dissipative; in this case when $Q<0$, system (21) is also stable.

Proof. For system (21), the time derivative of storage function $V\left(x_{L}(t), t\right)$ along the trajectory of system (21) is given as

$$
\begin{aligned}
\dot{V}\left(x_{L}(t)\right)= & \dot{x}_{L}^{\mathrm{T}}(t) P x_{L}(t)+x_{L}^{\mathrm{T}}(t) P \dot{x}_{L}(t) \\
= & \left(A_{L} x_{L}(t)-N_{L} y_{\mathrm{ref}}(t)\right)^{\mathrm{T}} P x_{L}(t) \\
& +x_{L}^{\mathrm{T}}(t) P\left(A_{L} x_{L}(t)-N_{L} y_{\mathrm{ref}}(t)\right)
\end{aligned}
$$

then we can know

$$
\begin{aligned}
\dot{V}\left(x_{L}\right. & (t))-r\left(y_{\text {ref }}(t), e(t)\right) \\
= & \left(A_{L} x_{L}(t)-N_{L} y_{\text {ref }}(t)\right)^{\mathrm{T}} P x_{L}(t) \\
& +x_{L}^{\mathrm{T}}(t) P\left(A_{L} x_{L}(t)-N_{L} y_{\mathrm{ref}}(t)\right) \\
& -\left(C_{L} x_{L}(t)-y_{\text {ref }}(t)\right)^{\mathrm{T}} \mathrm{Q}\left(C_{L} x_{L}(t)-y_{\text {ref }}(t)\right) \\
& -2\left(C_{L} x_{L}(t)-y_{\mathrm{ref}}(t)\right)^{\mathrm{T}} S y_{\mathrm{ref}}(t)-y_{\mathrm{ref}}^{\mathrm{T}}(t) R y_{\mathrm{ref}}(t) \\
= & \left(A_{L} x_{L}(t)-N_{L} y_{\mathrm{ref}}(t)\right)^{\mathrm{T}} P x_{L}(t) \\
& +x_{L}^{\mathrm{T}}(t) P\left(A_{L} x_{L}(t)-N_{L} y_{\mathrm{ref}}(t)\right) \\
& -\left(C_{L} x_{L}(t)-y_{\mathrm{ref}}(t)\right)^{\mathrm{T}} \mathrm{Q}\left(C_{L} x_{L}(t)-y_{\mathrm{ref}}(t)\right) \\
& -\left(C_{L} x_{L}(t)-y_{\mathrm{ref}}(t)\right)^{\mathrm{T}} S y_{\mathrm{ref}}(t) \\
& -y_{\mathrm{ref}}^{\mathrm{T}}(t) S^{\mathrm{T}}\left(C_{L} x_{L}(t)-y_{\mathrm{ref}}(t)\right)-y_{\mathrm{ref}}^{\mathrm{T}}(t) R y_{\mathrm{ref}}(t)
\end{aligned}
$$




$$
\begin{aligned}
& =\left[\begin{array}{c}
x_{L}(t) \\
y_{\text {ref }}(t)
\end{array}\right]^{\mathrm{T}} \\
& \times\left[\begin{array}{cc}
A_{L}^{\mathrm{T}} P+P A_{L}-C_{L}^{\mathrm{T}} Q C_{L} & -P N_{L}+C_{L}^{\mathrm{T}} Q-C_{L}^{\mathrm{T}} S \\
-N_{L}^{\mathrm{T}} P+Q C_{L}-S^{\mathrm{T}} C_{L} & -Q+S+S^{\mathrm{T}}-R
\end{array}\right] \\
& \times\left[\begin{array}{c}
x_{L}(t) \\
y_{\text {ref }}(t)
\end{array}\right] \text {. }
\end{aligned}
$$

Therefore, if LMIs (23) have a feasible solution $P$, it is from Definition 1 that tracking system (21) is dissipative. have

It is noted that when system (21) is strictly dissipative, we

$$
A_{L}^{\mathrm{T}} P+P A_{L}-C_{L}^{\mathrm{T}} Q C_{L}<0 .
$$

This together with $Q<0$ implies that

$$
A_{L}^{\mathrm{T}} P+P A_{L}<0 .
$$

Then, by the Lyapunov stability theory in [24], it can be verified that system (21) is stable. This completes the proof.

Now, we consider the dissipative output tracking statefeedback controller for the linear time-delay system (5) with the following form:

$$
\begin{aligned}
u(t) & =K_{1} x_{L}(t)+K_{2} x_{L}(t-d) \\
& =K_{11} x(t)+K_{12} q(t)+K_{21} x(t-d)+K_{22} q(t-d),
\end{aligned}
$$

where $K_{1}=\left[\begin{array}{ll}K_{11} & K_{12}\end{array}\right], K_{2}=\left[\begin{array}{ll}K_{21} & K_{22}\end{array}\right]$ are the controller gains to be determined. Then the closed-loop system can be obtained by applying the above controller to system (5), which is described as

$$
\begin{aligned}
\dot{x}_{L}(t)= & \left(A_{L}+B_{L} K_{1}\right) x_{L}(t)+\left(A_{L d}+B_{L} K_{2}\right) x_{L}(t-d) \\
& -N_{L} y_{\text {ref }}(t), \\
e(t)= & \left(C_{L}+D K_{1}\right) x_{L}(t)+\left(C_{L d}+D K_{2}\right) x_{L}(t-d) \\
& -y_{\text {ref }}(t), \\
x_{L}(t)= & {\left[\begin{array}{c}
\eta(t) \\
0
\end{array}\right]=\eta(t), \quad t \in[-d, 0] . }
\end{aligned}
$$

For the augmented system (5), we present a controller design method in the following theorem which guarantees that the closed-loop system (29) is both strictly dissipative and stable.

Theorem 7. For the linear time-delay tracking system (29) and the given supply rate (8) with $Q<0$, if the following LMIs

$$
\begin{gathered}
\\
{\left[\begin{array}{cccc}
\Xi_{11} & \Xi_{12} & \Xi_{13} & \left(C_{L} X+D W_{1}\right)^{\mathrm{T}} \\
* & \Xi_{22} & \Xi_{23} & 0 \\
* & * & -M & \left(C_{L d} X+D W_{2}\right)^{\mathrm{T}} \\
* & * & * & Q^{-1}
\end{array}\right]<0}
\end{gathered}
$$

have feasible solutions $W_{1}, W_{2}, X$, and $M$, then the closedloop system (29) is strictly dissipative and stable. Moreover, if $W_{1}^{*}, W_{2}^{*}$, and $X^{*}$ are the feasible solutions of (30), then the desired state-feedback controller is

$$
\begin{aligned}
u(t) & =K_{1} x_{L}(t)+K_{2} x_{L}(t-d) \\
& =W_{1}^{*}\left(X^{*}\right)^{-1} x_{L}(t)+W_{2}^{*}\left(X^{*}\right)^{-1} x_{L}(t-d) .
\end{aligned}
$$

The "*" in LMIs denotes the terms that can be induced by symmetric block in a matrix, where

$$
\begin{gathered}
\Xi_{11}=\left(A_{L} X+B_{L} W_{1}\right)+\left(A_{L} X+B_{L} W_{1}\right)^{\mathrm{T}}+M, \\
\Xi_{12}=-N_{L}+\left(C_{L} X+D W_{1}\right)^{\mathrm{T}} Q-\left(C_{L} X+D W_{1}\right)^{\mathrm{T}} S, \\
\Xi_{13}=A_{L d} X+B_{L} W_{2}, \\
\Xi_{22}=-Q+S+S^{\mathrm{T}}-R, \\
\Xi_{23}=Q\left(C_{L d} X+D W_{2}\right)-S^{\mathrm{T}}\left(C_{L d} X+D W_{2}\right) .
\end{gathered}
$$

Proof. According to Theorem 5, for the given supply rate (8) and the closed-loop system (29), if the following LMIs

$$
\begin{gathered}
P=P^{\mathrm{T}}>0 \\
{\left[\begin{array}{ccc}
\bar{A}_{L}^{\mathrm{T}} P+P \bar{A}_{L}+V-\bar{C}_{L}^{\mathrm{T}} \mathrm{Q} \bar{C}_{L} & -P N_{L}+\bar{C}_{L}^{\mathrm{T}} \mathrm{Q}-\bar{C}_{L}^{\mathrm{T}} S & P \bar{A}_{L d}-\overline{\mathrm{C}}_{L}^{\mathrm{T}} \mathrm{Q} \bar{C}_{L d} \\
* & -\mathrm{Q}+S+S^{\mathrm{T}}-R & \mathrm{Q} \bar{C}_{L d}-S^{\mathrm{T}} \bar{C}_{L d} \\
* & * & -V-\bar{C}_{L d}^{\mathrm{T}} \mathrm{Q} \bar{C}_{L d}
\end{array}\right]<0}
\end{gathered}
$$

have feasible solutions $P, V$, then system (29) is strictly dissipative and stable, where

$$
\begin{array}{cc}
\bar{A}_{L}=A_{L}+B_{L} K_{1}, & \bar{C}_{L}=C_{L}+D K_{1}, \\
\bar{A}_{L d}=A_{L d}+B_{L} K_{2}, & \bar{C}_{L d}=C_{L d}+D K_{2} .
\end{array}
$$

Premultiply and postmultiply $\operatorname{diag}\left\{P^{-1}, I, P^{-1}\right\}$ to the matrix inequality (33), and assume that $X=P^{-1}, W_{1}=K_{1} X$, $W_{2}=K_{2} X, M=X V X$, then using Schur complement lemma, the LMI (30), which is equivalent to the matrix inequality (33), can be obtained. Therefore, if the LMI (30) has feasible solutions $W_{1}^{*}, W_{2}^{*}$, and $X^{*}$, then $u(t)=K_{1} x_{L}(t)+$ $K_{2} x_{L}(t-d)=W_{1}^{*}\left(X^{*}\right)^{-1} x_{L}(t)+W_{2}^{*}\left(X^{*}\right)^{-1} x_{L}(t-d)$ is the desired dissipative state-feedback controller. This completes the proof.

Remark 8. It is noted that Theorem 7 provides a controller design method such that the resultant closed-loop system (29) is not only strictly dissipative but also stable, which can be more desirable to be obtained.

Remark 9. It should be pointed out that the designed controller of the form (28) is more general than some existing results. When $K_{2}=0$, the controller (28) reduces to the instant state feedback, while in case of $K_{1}=0$, it is just a retarded state feedback. 
Similarly to Theorem 6, in the absence of time delay, the dissipative output tracking state-feedback controller (28) reduces to the controller below

$$
u(t)=K x_{L}(t)=K_{1} x(t)+K_{2} q(t)
$$

where $K=\left[\begin{array}{ll}K_{1} & K_{2}\end{array}\right]$ is the controller gain to be determined, then the closed-loop system (29) is simplified to the following system:

$$
\begin{gathered}
\dot{x}_{L}(t)=\left(A_{L}+B_{L} K\right) x_{L}(t)-N_{L} y_{\text {ref }}(t), \\
e(t)=\left(C_{L}+D K\right) x_{L}(t)-y_{\text {ref }}(t) .
\end{gathered}
$$

Consequently, the following design method can be obtained.

Theorem 10. For system (36) and the given supply rate (8) with $Q<0$, if the following LMIs

$$
\begin{gathered}
X=X^{\mathrm{T}}>0 \\
{\left[\begin{array}{ccc}
\Xi_{11} & \Xi_{12} & \left(C_{L} X+D W\right)^{\mathrm{T}} \\
& -Q+S+S^{\mathrm{T}}-R & 0 \\
* & * & Q^{-1}
\end{array}\right]<0}
\end{gathered}
$$

have feasible solutions $W, X$, then the closed-loop system (36) is strictly dissipative and stable. If $W^{*}, X^{*}$ are the feasible solution of (37), then the desired state-feedback controller is $u(t)=$ $K x_{L}(t)=W^{*}\left(X^{*}\right)^{-1} x_{L}(t)$. The "*" in LMIs denotes the terms that can be induced by symmetric block in a matrix, where

$$
\begin{gathered}
\Xi_{11}=\left(A_{L} X+B_{L} W\right)+\left(A_{L} X+B_{L} W\right)^{\mathrm{T}} \\
\Xi_{12}=-N_{L}+\left(C_{L} X+D W\right)^{\mathrm{T}} Q-\left(C_{L} X+D W\right)^{\mathrm{T}} S .
\end{gathered}
$$

Proof. According to Theorem 6, for the given supply rate (8) and system (36), if LMIs

$$
\begin{gathered}
P=P^{\mathrm{T}}>0 \\
{\left[\begin{array}{cc}
\bar{A}_{L}^{\mathrm{T}} P+P \bar{A}_{L}-\bar{C}_{L}^{\mathrm{T}} Q \bar{C}_{L} & -P N_{L}+\bar{C}_{L}^{\mathrm{T}} \mathrm{Q}-\bar{C}_{L}^{\mathrm{T}} S \\
-N_{L}^{\mathrm{T}} P+Q \bar{C}_{L}-S^{\mathrm{T}} \bar{C}_{L} & -Q+S+S^{\mathrm{T}}-R
\end{array}\right]<0}
\end{gathered}
$$

have a feasible solution $P$, then system (36) is strictly dissipative and stable, where

$$
\bar{A}_{L}=A_{L}+B_{L} K, \quad \bar{C}_{L}=C_{L}+D K .
$$

Pre-multiply and post-multiply the matrix inequality (39) with $\operatorname{diag}\left\{P^{-1}, I\right\}$, and assume that $X=P^{-1}, W=K X$. According to Schur complement lemma, LMI (37), which is equivalent to the matrix inequality (39), can be obtained. Therefore, if the LMI (37) has feasible solutions $W^{*}, X^{*}$, then $u(t)=K x_{L}(t)=W^{*}\left(X^{*}\right)^{-1} x_{L}(t)$ is the dissipative statefeedback controller. This completes the proof.

\section{Numerical Simulation}

In order to demonstrate the applicability and effectiveness of the developed method, we present a numerical simulation example. Consider system (1) with the following data:

$$
\begin{array}{ccc}
A=\left[\begin{array}{cc}
-2 & 0 \\
1 & -3
\end{array}\right], & A_{d}=\left[\begin{array}{ll}
0 & 0 \\
0 & 0
\end{array}\right], & C=\left[\begin{array}{ll}
1 & -1
\end{array}\right], \\
C_{d}=\left[\begin{array}{ll}
0 & 0
\end{array}\right], & B=\left[\begin{array}{c}
-1 \\
1
\end{array}\right], & D=3.2 .
\end{array}
$$

Then, from (5), we can have the augmented system with the following data:

$$
\begin{gathered}
A_{L}=\left[\begin{array}{ccc}
-2 & 0 & 0 \\
1 & -3 & 0 \\
1 & -1 & 0
\end{array}\right], \quad A_{L d}=\left[\begin{array}{lll}
0 & 0 & 0 \\
0 & 0 & 0 \\
0 & 0 & 0
\end{array}\right], \\
B_{L}=\left[\begin{array}{c}
-1 \\
1 \\
3.2
\end{array}\right], \quad C_{L}=\left[\begin{array}{lll}
1 & -1 & 0
\end{array}\right], \quad C_{L d}=\left[\begin{array}{lll}
0 & 0 & 0
\end{array}\right], \\
N_{L}=\left[\begin{array}{l}
0 \\
0 \\
1
\end{array}\right], \quad D=3.2,
\end{gathered}
$$

and the parameters in supply rate are

$$
Q=-1, \quad S=0, \quad R=1.056 .
$$

Solving the LMIs (37) in Theorem 10 by using the LMI toolbox in MATLAB, we can get

$$
\begin{aligned}
& X=\left[\begin{array}{ccc}
0.2754 & 0.0238 & 0.1002 \\
0.0238 & 0.1965 & -0.0501 \\
0.1002 & -0.0501 & 0.3400
\end{array}\right], \\
& W=\left[\begin{array}{lll}
-0.0786 & 0.0540 & -0.3007
\end{array}\right] .
\end{aligned}
$$

Hence, the following dissipative state-feedback controller can be obtained:

$$
u(t)=K x_{L}(t)=\left[\begin{array}{lll}
-0.0339 & 0.0440 & -0.8879
\end{array}\right] x_{L}(t) .
$$

Assume that the reference system has the following form:

$$
\begin{gathered}
\dot{x}_{\text {ref }}(t)=-10 x_{\text {ref }}(t)+r(t) \\
y_{\text {ref }}(t)=0.5 x_{\text {ref }}(t)
\end{gathered}
$$

(1) choose $r(t)=5 \cos (t)$, when $x_{L}(0)=\left[\begin{array}{lll}0 & 0 & 0\end{array}\right]^{\mathrm{T}}$, $x_{\text {ref }}(0)=0$, output tracking trajectory is shown in Figure 1; when $x_{L}(0)=\left[\begin{array}{lll}1 & 0 & 1\end{array}\right]^{\mathrm{T}}, x_{\text {ref }}(0)=1$, output tracking trajectory is shown in Figure 2;

(2) choose $r(t)=0$, when $x_{L}(0)=\left[\begin{array}{lll}0 & 0 & 0\end{array}\right]^{\mathrm{T}}, x_{\text {ref }}(0)=$ 0 , output tracking trajectory is shown in Figure 3; when $x_{L}(0)=\left[\begin{array}{lll}1 & 0 & 1\end{array}\right]^{\mathrm{T}}, x_{\text {ref }}(0)=1$, output tracking trajectory is shown in Figure 4; 


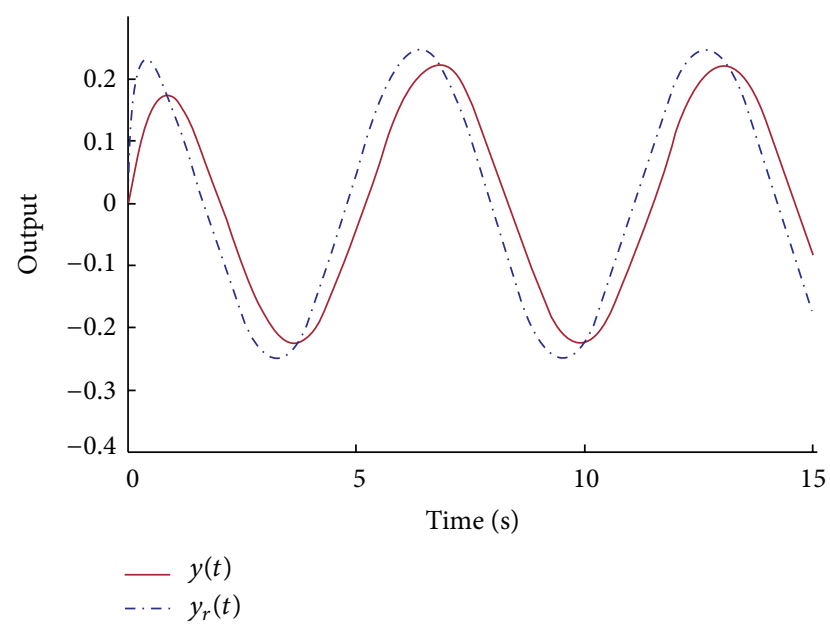

FIGURE 1: System output tracking trajectory with zero initial condition.

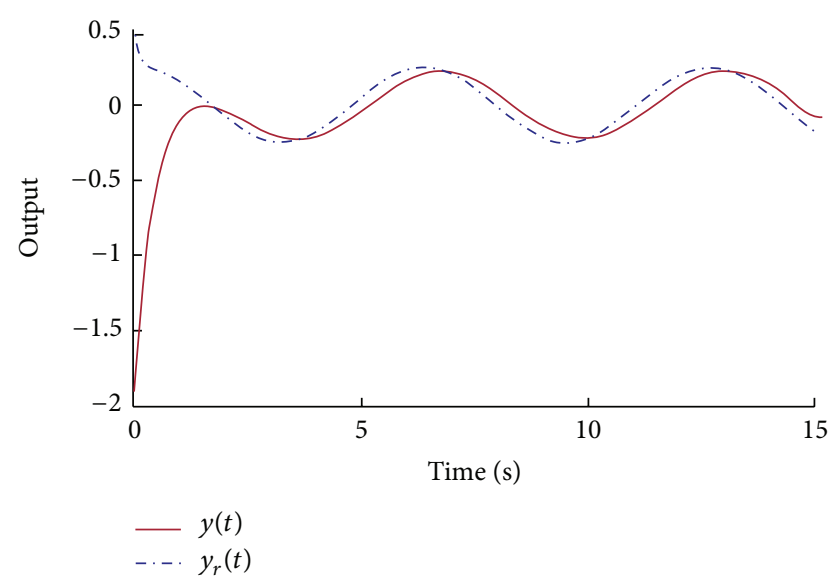

FIGURE 2: System output tracking trajectory with nonzero initial condition.

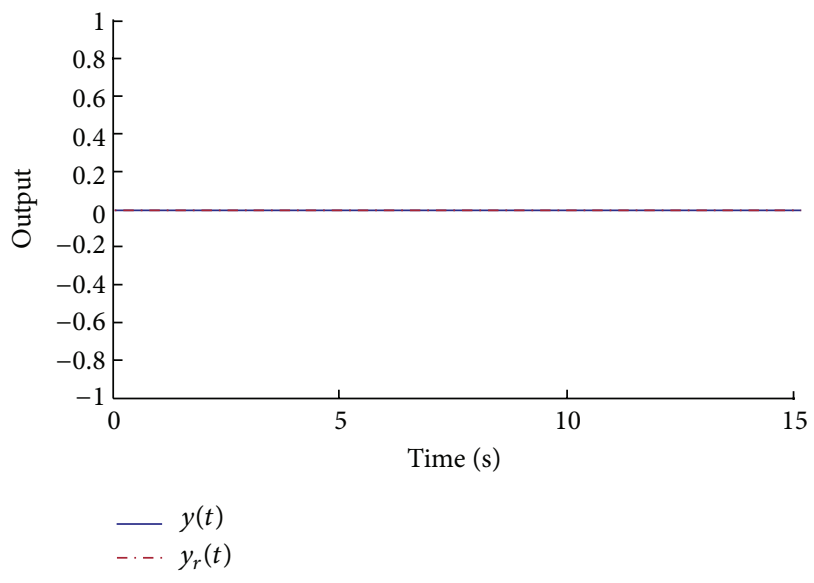

FIGURE 3: System output tracking trajectory with zero initial condition.

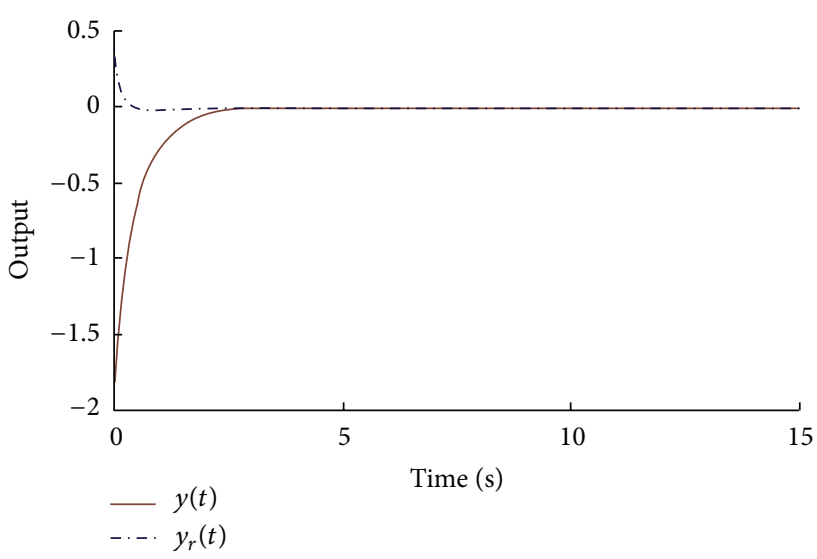

FIGURE 4: System output tracking trajectory with nonzero initial condition.

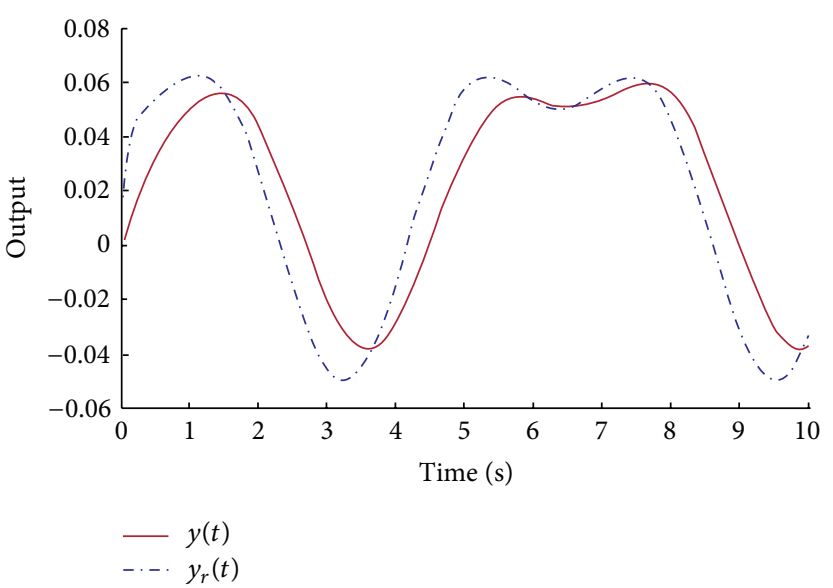

FIGURE 5: System output tracking trajectory with zero initial condition.

(3) choose $r(t)=(\sin (t))^{2}+\cos (t)$, when $x_{L}(0)=$ $\left[\begin{array}{lll}0 & 0 & 0\end{array}\right]^{\mathrm{T}}, x_{\text {ref }}(0)=0$, output tracking trajectory is shown in Figure 5; when $x_{L}(0)=\left[\begin{array}{lll}1 & 0 & 1\end{array}\right]^{\mathrm{T}}, x_{\text {ref }}(0)=$ 1 , output tracking trajectory is shown in Figure 6.

According to Figures 1-6, we find that the output vector can approach the reference output vector as closely as possible; it is obvious that our proposed method is very effective.

\section{Conclusion}

By the example and the theorems, we can draw the following conclusions. Compared with most output tracking control methods, this paper realizes the output tracking performance by stable and dissipative controllers. The theorems are computationally simple and practically effective. Meanwhile, there still exist many problems that need to be solved. The tracking performance should be further investigated, and in 


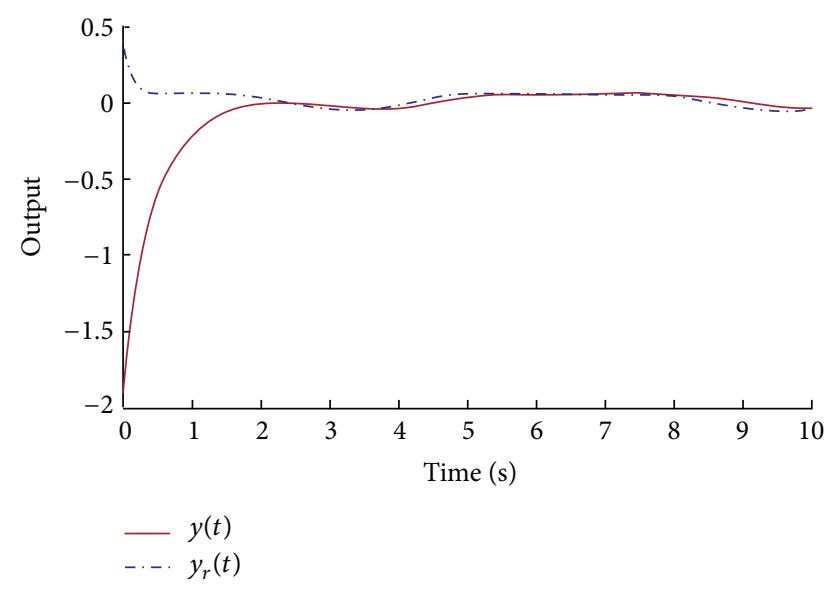

FIGURE 6: System output tracking trajectory with nonzero initial condition.

the presence of uncertainties, the robust dissipative control for the systems is also worthy to be studied.

\section{Acknowledgment}

This work was partially supported by The Natural Science Foundation of China under Grants nos. 60974004, 61273003, and 61273008 , respectively.

\section{References}

[1] P. Martin, S. Devasia, and B. Paden, "A different look at output tracking: control of a VTOL aircraft," Automatica, vol. 32, no. 1, pp. 101-107, 1996.

[2] E. Kim, "Output feedback tracking control of robot manipulators with model uncertainty via adaptive fuzzy logic," IEEE Transactions on Fuzzy Systems, vol. 12, no. 3, pp. 368-378, 2004.

[3] F. Zhang, D. M. Dawson, M. S. de Queiroz, and W. E. Dixon, "Global adaptive output feedback tracking control of robot manipulators," IEEE Transactions on Automatic Control, vol. 45, no. 6, pp. 1203-1208, 2000.

[4] K. D. Do, Z.-P. Jiang, and J. Pan, "A global output-feedback controller for simultaneous tracking and stabilization of unicycletype mobile robots," IEEE Transactions on Robotics and Automation, vol. 20, no. 3, pp. 589-594, 2004.

[5] Q. L. Zhang, C. Liu, and X. Zhang, Complexity, analysis and control of singular biological systems, vol. 421 of Lecture Notes in Control and Information Sciences, Springer, London, UK, 2012.

[6] Z.-P. Jiang, "Decentralized and adaptive nonlinear tracking of large-scale systems via output feedback," IEEE Transactions on Automatic Control, vol. 45, no. 11, pp. 2122-2128, 2000.

[7] B. Chen, X. Liu, and S. Tong, "Adaptive fuzzy output tracking control of MIMO nonlinear uncertain systems," IEEE Transactions on Fuzzy Systems, vol. 15, no. 2, pp. 287-300, 2007.

[8] S. Tong, T. Wang, and J. T. Tang, "Fuzzy adaptive output tracking control of nonlinear systems," Fuzzy Sets and Systems, vol. 111, no. 2, pp. 169-182, 2000.

[9] K. S. Narendra and N. O. Oleng, "Exact output tracking in decentralized adaptive control systems," IEEE Transactions on Automatic Control, vol. 47, no. 2, pp. 390-395, 2002.
[10] C. Edwards and S. K. Spurgeon, "Robust output tracking using a sliding-mode controller/observer scheme," International Journal of Control, vol. 64, no. 5, pp. 967-983, 1996.

[11] Z.-H. Li, T.-Y. Chai, C. Y. Wen, and C.-B. Soh, "Robust output tracking for nonlinear uncertain systems," Systems \& Control Letters, vol. 25, no. 1, pp. 53-61, 1995.

[12] C. Hua, X. Guan, and P. Shi, "Robust output feedback tracking control for time-delay nonlinear systems using neural network," IEEE Transactions on Neural Networks, vol. 18, no. 2, pp. 495505, 2007.

[13] C. Lin, Q.-G. Wang, and T. H. Lee, " $H_{\infty}$ output tracking control for nonlinear systems via T-S fuzzy model approach," IEEE Transactions on Systems, Man, and Cybernetics B, vol. 36, no. 2, pp. 450-457, 2006.

[14] H. Gao and T. Chen, "Network-based $H_{\infty}$ output tracking control," IEEE Transactions on Automatic Control, vol. 53, no. 3, pp. 655-667, 2008.

[15] Y. Zhang, Q. L. Zhang, and L.-C. Zhao, “Tracking control of a chaotic SIR epidemic model," Journal of Biomathematics, vol. 23, no. 3, pp. 457-462, 2008.

[16] Y. Zhang, Q. L. Zhang, L.-C. Zhao, and P.-Y. Liu, "Tracking control of chaos in singular biological economy systems," Journal of Northeastern University, vol. 28, no. 2, pp. 157-164, 2007.

[17] N. Yi, Analysis and control problem research for several epidemic dynamic systems [Ph.D. thesis], Northeastern University, China.

[18] J. C. Willems, "Dissipative dynamical systems. I. General theory," Archive for Rational Mechanics and Analysis, vol. 45, pp. 321-351, 1972.

[19] J. C. Willems, "Dissipative dynamical systems. II. Linear systems with quadratic supply rates," Archive for Rational Mechanics and Analysis, vol. 45, pp. 352-393, 1972.

[20] Z. Tan, Y. C. Soh, and L. Xie, "Dissipative control for linear discrete-time systems," Automatica, vol. 35, no. 9, pp. 1557-1564, 1999.

[21] S. Xie, L. Xie, and C. E. de Souza, "Robust dissipative control for linear systems with dissipative uncertainty," International Journal of Control, vol. 70, no. 2, pp. 169-191, 1998.

[22] H. Zhang, Z.-H. Guan, and G. Feng, "Reliable dissipative control for stochastic impulsive systems," Automatica, vol. 44, no. 4, pp. 1004-1010, 2008.

[23] S. Boyd, L. El Ghaoui, E. Feron, and V. Balakrishnan, Linear Matrix Inequalities in System and Control Theory, vol. 15 of SIAM Studies in Applied Mathematics, SIAM, Philadelphia, Pa, USA, 1994.

[24] S. Y. Xu and J. Lam, Robust Control and Filtering of Singular Systems, vol. 332 of Lecture Notes in Control and Information Sciences, Springer, Berlin, Germany, 2006. 


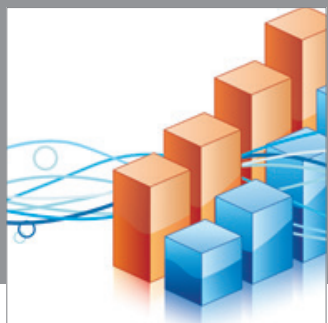

Advances in

Operations Research

mansans

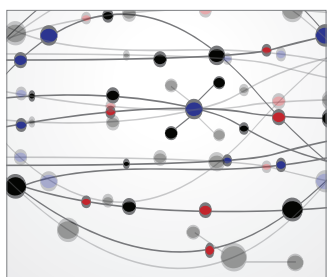

The Scientific World Journal
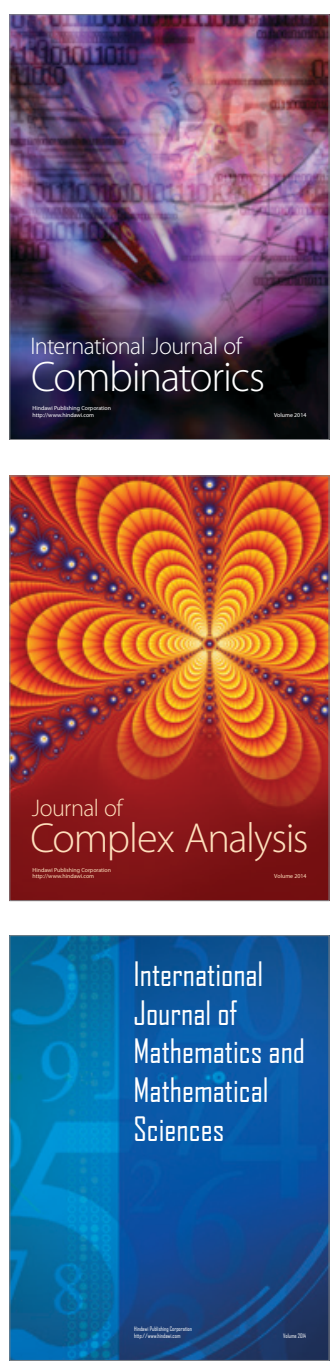
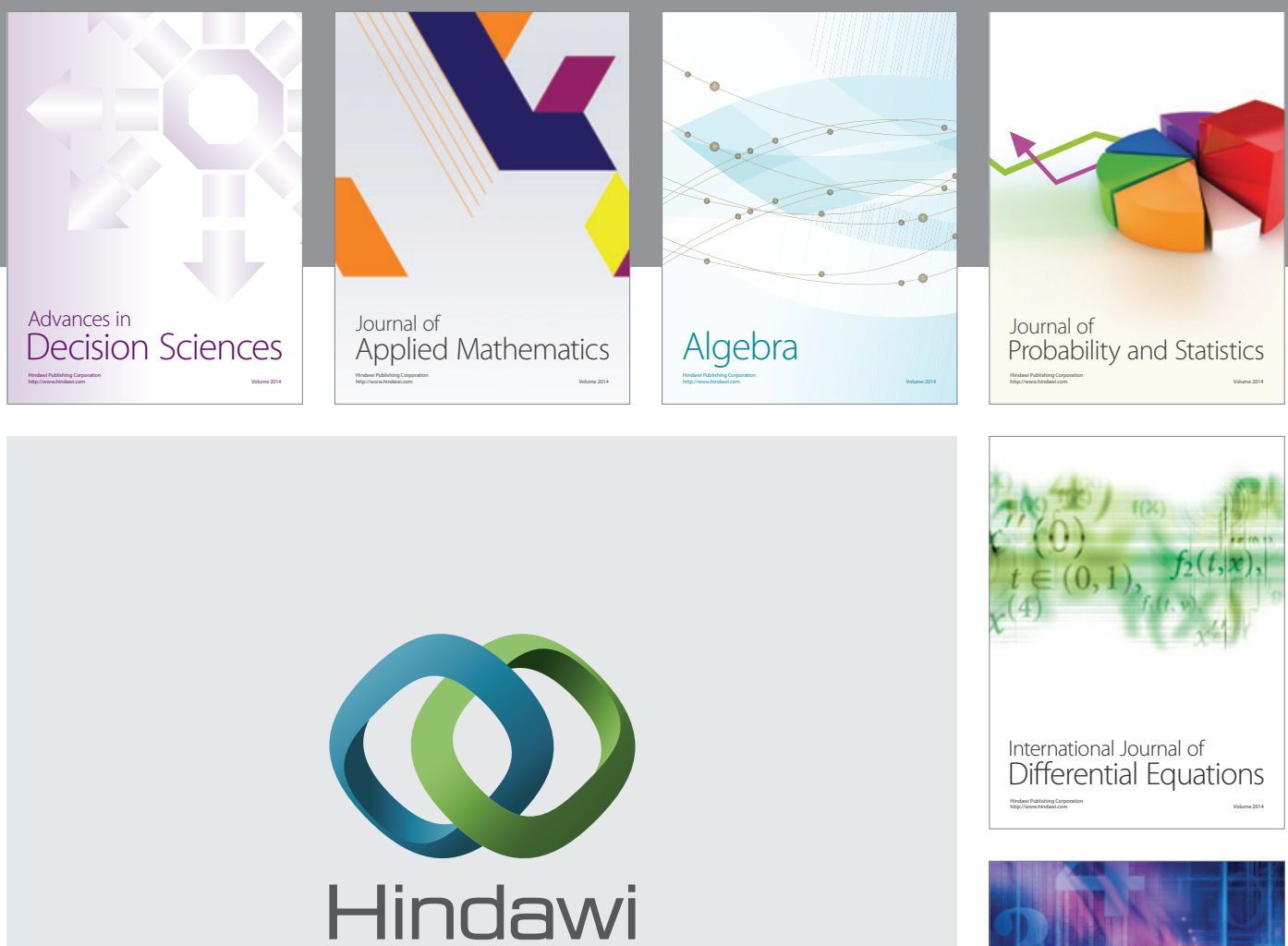

Submit your manuscripts at http://www.hindawi.com
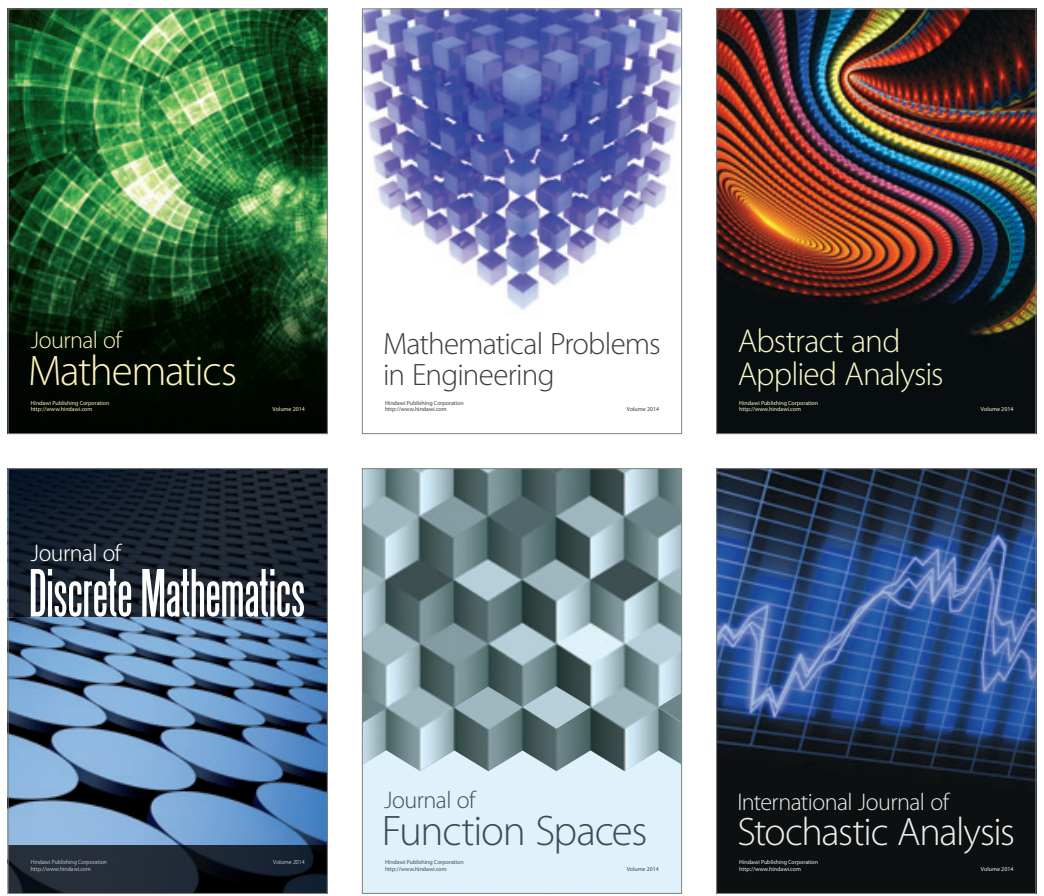

Journal of

Function Spaces

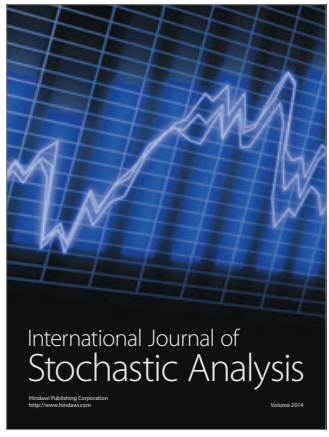

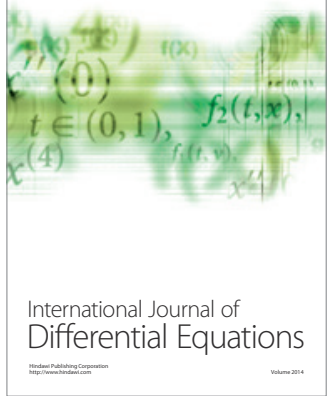
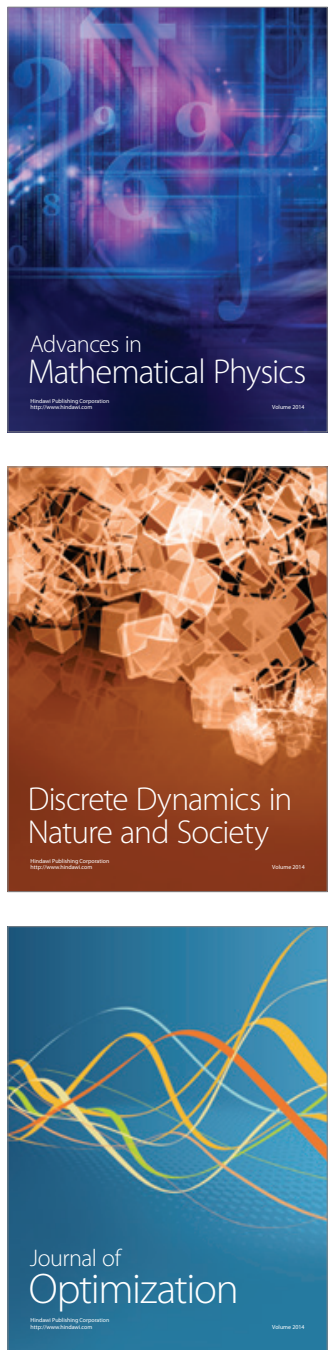